\title{
Design and Implementation of A Content-based Gift Recommender System for Patient-visit
}

\author{
Yang Yu \\ School of Software Engineering of \\ Beijing University of Posts and Telecommunications \\ Beijing, China \\ fishoutsider@gmail.com
}

\begin{abstract}
When visiting a patient, it's difficult to choose a useful gift for the patient. Moreover, we often send some useless gifts. This system is able to solve the problem. It can recommend gifts according to the doctors' suggestions and the patient's basic information. We created a mathematical model for this problem considering the type of disease and the patient's personal information. Then it uses the content-based recommendation algorithm to provide different types of gifts for the visitors. Next we validated the performance through computing the time complexity. Finally, we summarized what we have done and put forward how to improve in the future.
\end{abstract}

Keywords- classification; personality; recommendation; patient-visitor; gift

\section{INTRODUCTION}

In interpersonal communication, we often give each other gifts. Choosing a gift has become a universal problem, especially when given to patients. We want to pick something which is good for the rehabilitation of patients. And it'll be perfect if the patient loves it. We want to help find the right gift. At first, we should consider the type of disease. Then we need discover the patient's favorite things. At last, the system can consider these aspects and make recommendations according to the type of items.

\section{METHODS}

\section{A. Fresh patient recommendation}

If the patient just joined the system, we don't know his favorite. In this case, there is no way to make recommendations based on his preferences. At this time, we should consider the disease, age, gender to make a recommendation. Moreover, taking into account the practically of the gift, a suitable nutrition will be a good choice. So for these patients, we can screen through the patient's demand for all kinds of nutrients. Meanwhile, we can find the patients who have a similar condition and take their data as a reference to make a recommendation.

The essential nutrients in body can be grouped into six categories: proteins, fats, carbohydrates and trace elements, vitamins, minerals and water.

\author{
YuHui Wang \\ School of Materials Engineering of \\ Fujian University of Agriculture and Forestry \\ Fujian, China \\ w825988471@yahoo.com.tw
}

In this system, we choose protein, vitamin and mineral according to the supplements' classifications on the market. The system calculates the demand index of these three indicators separately and then finds the highest to recommend.

Demand index calculated as follows:

$$
\mathrm{DI}=\frac{\mathrm{I}_{\text {age }} \times \mathrm{L}_{\text {age }}+\mathrm{I}_{\text {sex }} \times \mathrm{L}_{\text {sex }}}{\mathrm{A}_{\mathrm{v}}}+\mathrm{I}_{\text {illness }}
$$

In formula (1), use DI to represent demand index, use Iage to represent effect index of age on demand index, use $A_{v}$ to represent the average demand for all, use Lage to represent the patient's age level.

Effect index of age calculated as follows:

$$
I_{\text {age }}=\frac{\sqrt{\left(D_{c}-A_{v}\right)^{2}+\left(D_{t}-A_{v}\right)^{2}+\left(D_{e}-A_{v}\right)^{2}}}{3}
$$

Effect index of sex calculated as follows:

$$
\mathrm{I}_{\mathrm{sex}}=\frac{\sqrt{\left(D_{f}-\mathrm{A}_{\mathrm{v}}\right)^{2}+\left(D_{m}-\mathrm{A}_{\mathrm{v}}\right)^{2}}}{2}
$$

In formula (2), $D_{c}, D_{t}, D_{e}$ represent the average demand of children, young man and elder.

In formula (3), $D_{f}, D_{m}$ represent the average demand of woman and man.

Age and sex are two important effect factors. Due to the different evaluation criteria for each nutrient, we can't use the value to measure simply. But we can use the percentage of value's change to measure. So we do normalization between Iage and Isex. Then we can modify the formula (1) to formula (4) as follows:

$$
\mathrm{DI}=\frac{\mathrm{I}_{\mathrm{a}} \times \mathrm{Lage}_{\text {ag }}+\mathrm{I}_{\mathrm{s}} \times \mathrm{L}_{\text {sex }}}{\mathrm{A}_{\mathrm{v}}}+\mathrm{I}_{\text {illness }}
$$


The type of disease has a huge impact on demand index. It's determinate that if there is a strong demand for each nutrient when the kind of disease is certain. So we can set different eigenvalues for each category of disease according to the doctors' recommendations. That is to say, we can determine whether there is a strong demand for a particular nutrient. If the answer is yes, the eigenvalue will be set to 1 . Otherwise it'll be 0 . When making recommendations, we should calculate demand index of protein, vitamin and mineral. And pick the highest to recommend.

\section{B. The patients with records}

1) Non-dietary recommendations: On top of these factors, we should also analyze patients' records collected from those who already have records to know patients' preference in order to provide recommendation on gifts of variety and individuality to visitors.

This part uses content-based recommendation algorithm.

a) Overall process: At first, we learn from the Amazon and other electronic business platforms for goods classification criteria. The gift roughly divided into several categories, and each category also includes the following sub-categories, and finally to a specific gift.

The general workflows of recommendation algorithm mainly includes: Data collection, Data preprocessing and Recommendation. This algorithm corresponds to the overall process shown in Fig. 1.

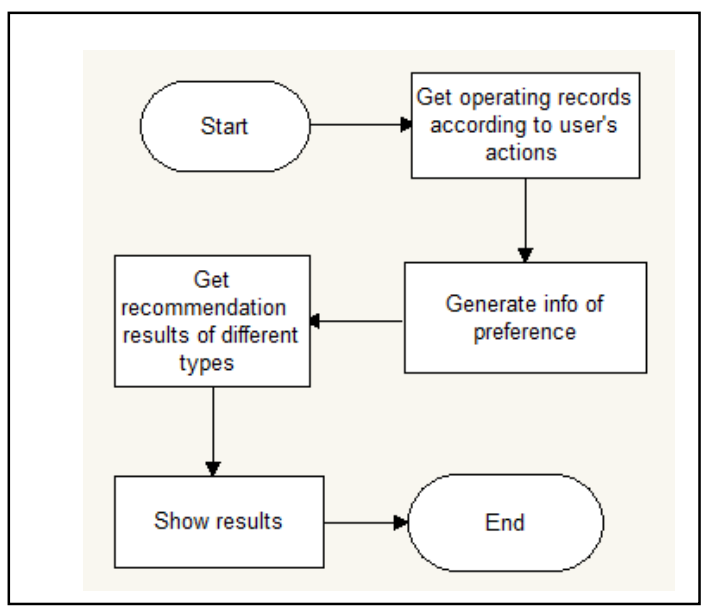

Fig. 1 Overall process

b) Data collection: Users' click behavior will be stored into Action table in the database after processed. Table I describes the structure of the Action table.

c) Preference model: This algorithm uses VSM as a formal representation method of the user's preference model. The system is able to make recommendations according to the data collected from the patients.

User model is represented as follows:

$$
M=\left(m_{\text {type }}, m_{\text {content }}, m_{\text {gift }}\right)
$$

In formula (5), $m_{\text {type }}, m_{\text {content }}, m_{\text {gift }}$ represent features of first-level index, second-level index and specific gifts. Then we integrate data results of three aspects to get preference result and recommend.

$$
m=(I, N)
$$

In formula (6), $I$ represents ID, $N$ represents numbers of clicks.

Preference value calculated as follows:

$$
I V=W_{t} \times N_{t}+W_{c} \times N_{c}+W_{g} \times N_{g}
$$

In formula (7), $I V$ represents preference value, $W_{t}$, $W_{c}, W_{g}$ represent weight of first-level index, second-level index and specific gifts, $N_{t}, N_{c}, N_{g}$ represent numbers of clicks separately.

TABLE I. STRUCTURE OF ACTION TABLE

\begin{tabular}{|l|l|c|}
\hline \multirow{2}{*}{ Name } & \multicolumn{2}{|c|}{ Attribute } \\
\cline { 2 - 3 } UserID & \multicolumn{1}{|c|}{ Capacity } & Description \\
\hline TypeID & Number(20) & UserID \\
\hline ContentID & Number(20) & First-level index ID \\
\hline GiftID & Number(20) & Gift ID \\
\hline TypeNum & Number(20) & Clicks of first-level index \\
\hline ContentNum & Number(20) & Clicks of second-level index \\
\hline GiftNum & Number(20) & Clicks of specific gift \\
\hline
\end{tabular}

It has been proved that we may achieve best effort of recommendation when $N_{t}=1, N_{c}=2, N_{c}=5$. (8).

Therefore the final preference value formula is formula

$$
I V=N_{t}+2 \times N_{c}+5 \times N_{g}
$$

Finally the system selects an index in the top two according to the preference result, and then selects the top three under the secondary level of the index respectively.Then it recommends two gifts from each subcategories.

2) Appetite Recommendation Process: In terms of the analyst on influence of gender, age, disease's type and preference result, the system can make recommendations for patients with records.

Recommendation value calculated as follows:

$$
R=W_{D I} \times \mathrm{DI}+W_{I V} \times I V
$$


In formula (9), $R$ represents recommendation value, $W_{D I}$ represents weight of demand index, $W_{I V}$ represents weight of preference value.

According to the recommendation value, recommend three proper items of food and nourishment.

\section{IMPLEMENTATION AND VERIFICATION}

\section{A. Nutrient requirement model}

1) Protein: Data for demand on protein classify by sex and age in Table II.

TABLE II. DEMAND VALUE

\begin{tabular}{|l|c|c|}
\hline \multirow{2}{*}{ Age } & \multicolumn{2}{|c|}{ Gender } \\
\cline { 2 - 3 } & Male & Female \\
\hline $1-23$ & 900 & 850 \\
\hline $24-50$ & 1000 & 1200 \\
\hline Above 50 & 1000 & 1200 \\
\hline
\end{tabular}

So we can calculate protein's demand index respectively in terms of age and gender. The modified formula as follows:

$$
\mathrm{DI}_{\text {protein }}=0.67 \times \mathrm{Lage}_{\mathrm{ag}}+0.33 \times \mathrm{L}_{\text {sex }}+\mathrm{I}_{\text {illness }}
$$

Level defined rules:

When data $=A_{v} \times(1 \pm 0.05)$, record is 0.5 . If data $>0.95 \times A_{v}$, record as 0 . Otherwise, record is 1 .

Protein level query table as Table III:

TABLE III. PROTEIN LEVEL

\begin{tabular}{|l|c|c|}
\hline \multirow{2}{*}{ Age } & \multicolumn{2}{|c|}{ Gender } \\
\cline { 2 - 3 } $1-23$ & Male & Female \\
\hline $24-50$ & 0 & 0 \\
\hline Above 50 & 0.5 & 1 \\
\hline
\end{tabular}

Computing of demand level: Lage is the summation of men and women in a certain age. Lsex is value from a certain gender.

For example: There is a 56-year-old man. His Lage is 1.5, Lsex is 1 .

2) Vitamin: Here we have statistics of vitamin A, vitamin C, Demand of vitamin calculated as follows:

$$
\mathrm{DI}_{\text {protein }}=0.06 \times \mathrm{L}_{\text {age }}+0.94 \times \mathrm{L}_{\text {sex }}+\mathrm{I}_{\text {illness }}
$$

Vitamin level query table as Table IV :
TABLE IV. VITAMIN LEVEL

\begin{tabular}{|l|c|c|}
\hline \multirow{2}{*}{ Age } & \multicolumn{2}{|c|}{ Gender } \\
\cline { 2 - 3 } & Male & Female \\
\hline $1-23$ & 0.5 & 0 \\
\hline $24-50$ & 1 & 0.5 \\
\hline Above 50 & 1 & 0 \\
\hline
\end{tabular}

3) Mineral: We have statistics of $\mathrm{Ca}, \mathrm{K}, \mathrm{Mg}$ as representatives.

Demand of mineral calculated as follows:

$$
\mathrm{DI}_{\text {protein }}=0.33 \times \mathrm{Lage}_{\mathrm{a}}+0.67 \times \mathrm{L}_{\text {sex }}+\text { Iillness }
$$

Mineral level query table as Table V :

TABLE V. MiNERAL LEVEL

\begin{tabular}{|l|c|c|}
\hline \multirow{2}{*}{ Age } & \multicolumn{2}{|c|}{ Gender } \\
\cline { 2 - 3 } & Male & Female \\
\hline $1-23$ & 0 & 0 \\
\hline $24-50$ & 1 & 1 \\
\hline Above 50 & 0.5 & 0 \\
\hline
\end{tabular}

\section{B. Real data validation}

We got the personal information of 67 patients under the patient's consent, including age, gender and type of disease. Then we consulted four doctors to judge which nutrient is needed as the first place for each patient. After that, we use SPSS for data analysis.

Actual results shown in Fig. 2; the results calculated by the system shown in Fig. 3.

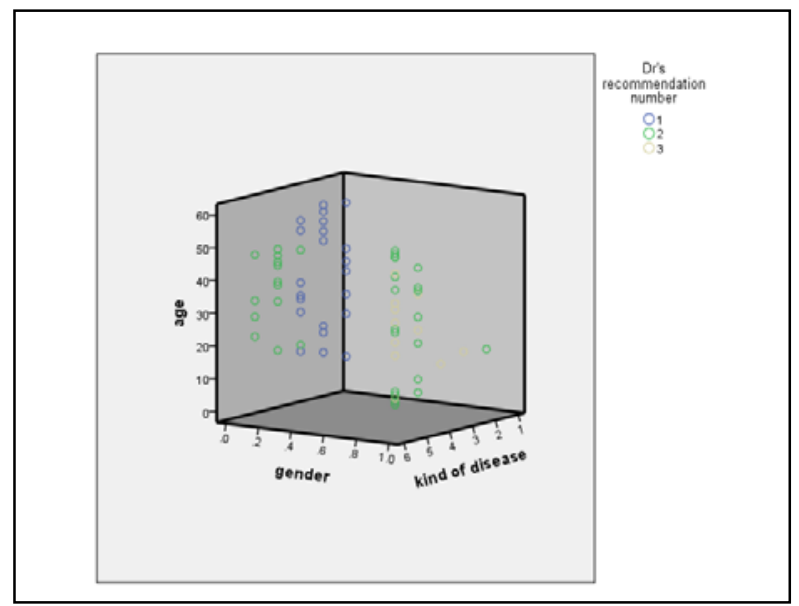

Fig. 2 Actual results 


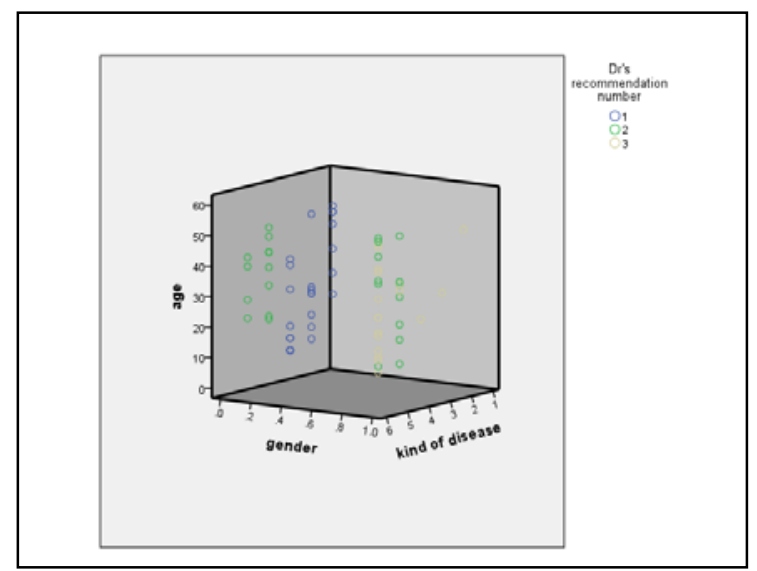

Fig. 3 Results calculated by the system

\section{CONCLUSION}

The system can make different recommendations for different users. It includes totally two user groups, new users and old users. Because we do not know new users' preferences, we recommend the nutrient which has the highest demand index to visitors, after calculating the demand index of protein, vitamin and mineral. Meanwhile we take full account of the old users' preference to make personalized recommendations besides nutrition. So we can provide the best solutions for the visitors.
Obviously, this recommended model is not perfect. There are still several issues:

1. Some other factors that may influence the recommendations are not included in the model. Such as, occupation, expertise and so on.

2. We should also consider the economic factor when recommending gifts. It's necessary to make different recommendations for different budgets.

3. There isn't evaluation criteria of users' satisfaction. So we can't measure the quality of recommended results.

\section{REFERENCES}

[1] Deng Ailin, Zhu Yangyong, Shi Bole, A Collaborative Filtering Recommendation Algorithm Based on Item Rating Prediction[J], JOURNAL OF SOFTWARE, 2003, 14 (9) : 162121628.

[2] Gao Jing, Ying JiKang, A Recommendation System Based on Artificial Immune System[J], COMPUTER TECHNOLOGY AND DEVELOPMENT, 2007, 17 (5) : 1802183.

[3] Li GuangYuan, Li WenJing, A clustering analysis method based on the similarities of the attributes(in Chinese) [J], COMPUTER AND INFORMATION TECHNOLOGY, 2002,4 (5) :530001.

[4] Wang WeiPing, Liu Ying, Recommendation Algorithm Based on Customer Behavior Locus[J], COMPUTER SYSTEMS \& APPLICATIONS, 2006, 15 (9) : 35238.

[5] Wang ZhiMei, Yang Fan, P2P Recommendation Algorithm Based on Hebbian Consistency Learning[J], COMPUTER ENGINEERING AND APPLICATIONS, 2006, 42 (36) : 1102113.

[6] Xu YiFeng, Xu QingYun, Liu XiaoPing, A Recommender Arithmetic Based on The Order of Time[J], COMPUTER SYSTEMS \& APPLICATIONS, 2006, 15 (10) : 22225, 29. 